\title{
ETHNOBOTANICAL RESEARCH IN GRAND TETON NATional PARK, Honoring The First PEOPLE AND Their Plant KNOWledge
}

\author{
$\gamma$ \\ EVELYN HILL \ DEPARTMENT OF BOTANY \\ UNIVERSITY OF WYOMING $\uparrow$ LARAMIE
}

\section{$\downarrow$ OBJECTIVES}

During the summer of 2004 I began the second phase of a three part study on ethnobotanical plants within the protected boundaries of Grand Teton National Park (GTNP) and the Rockefeller Jr. Memorial Parkway. Identification, location and collection of around 300 plant species used for food, medicine, utilitarian and ceremonial purposes by Shoshoni, Blackfoot, Crow, Ute, Arapahoe, Gosiute and Western Plains and Rocky Mountain Tribes, was and still is the goal of this project.

\section{$\downarrow$ METHODS}

The first phase, begun in Spring 2004 examined prehistoric, multi-tribal flora remains through archeological site reports, dated 1976 through 1993, and netted 28 sites in Grand Teton National Park from the Wyoming State Historic Preservation Office (SHPO), Laramie, Wyoming and the Midwest Archaeological Center, National Park Service, Lincoln, Nebraska (Table 1). Many of these archeological sites are located on the old shore line of Jackson Lake, and also scattered throughout GTNP. These reports yield information on botanical and pollen species, charcoal and hearth analyses, and flotation work if performed. Other historic records found on the uses of plant species in this biome came from many sources including librarians, friends, old bibliographies, early photographers, historians, natural fiber dyers, fellow herbalists, and a few phone calls from enthusiastic people wanting to share a rare book or pamphlet, having heard of this project.

\section{$\downarrow \quad$ RESULTS}

Collecting a specific set of plants entails knowing their habitat requirements, seasonal growth habits, local weather factors and the stages of flowering and seed maturation by species. By staggering collecting times into two week intervals, I was able to coincide collecting with plant growth habits. For the 42 days spent in the field the results include 39 families and 70 species to date and their identifications are tentative, not verified (Table 2). Some of the plants I have searched for and not yet found may never be found, due to extinction or historically incorrect identification. Plants could very possibly have been carried into the area by tribe visitation from long distances or traded for and, roasted and eaten, leaving only a few charred seeds behind as the evidence found in archaeological records.

\section{$\downarrow \quad$ CONCLUSIONS}

Further collecting of plant specimens is needed to complete the study's second phase. The harvesting of seeds into the fall will meet the third and final goal of providing identifiable macrofloral material for charring. Charring reproduces the seed content of hearths, creating a set of seeds for archaeological analytical comparison. These three 
phases will help create a basis for future archaeological, botanical and cultural investigations into the ethnobotany of Grand Teton National Park.

\section{$\uparrow \quad$ ADDENDUM}

Along with the three phase study, I brought the entire UW-NPS plant collection, located at the AMK Ranch, to the Rocky Mountain Herbarium for review, development and for the addition of species not contained in their comparative collection. Drs. Hank Harlow, Greg Brown, Ron Hartman and Ernie Nelson and I feel this is an important expansion of reference material for all researchers and disciplines.

\section{$\uparrow$ ACKNOWLEDGMENTS}

I am most appreciative of the enthusiastic interest and guidance of Drs. Judith Antell, Greg Brown and William Gribb; financial research support from the Wyoming Native Plant Society and the University of Wyoming-National Park Service (UWNPS); the facilities available for on-site research and the generous help of Rich Viola and the staff at the UW-NPS Research Center; Grand Teton National Park (GTNP) Service personnel including Sue Consolo-Murphy, Chief, Science \& Resource Management, for her interest and insights, Jacqueline St. Clair, Cultural Resource Office, for her dedication and encouragement of this project, Klara Varga, Botanist, and her staff for help with plant location. With sincere appreciation, I thank Lanie Thom, GTNP Shoshoni Life Interpretive, for our conversations about plant properties and inspirations on studying ethnotaxonomy.

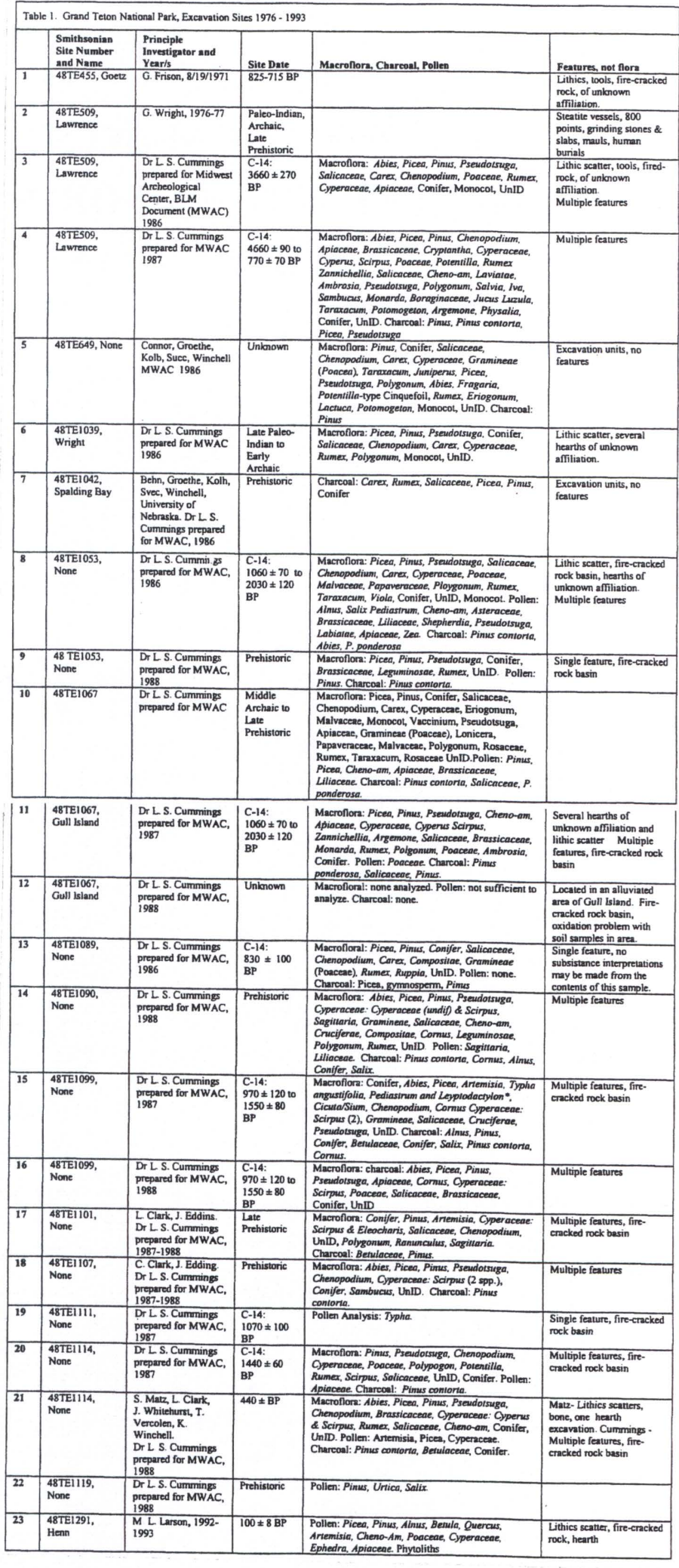


Table 2. Grand Teton National Park, Ethnobotanical Collection 2004. Evelyn Hill

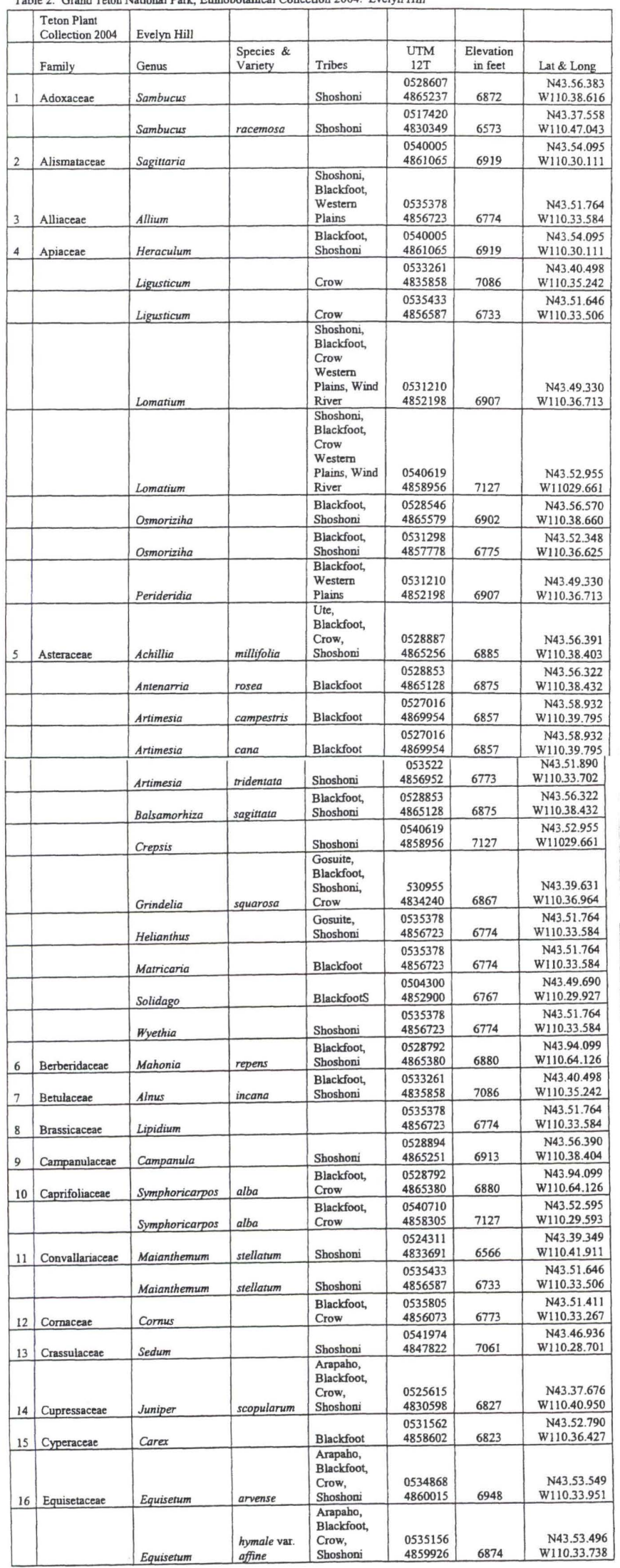

\begin{tabular}{|c|c|c|c|c|c|c|c|}
\hline 17 & Ericaceae & Arctostaphylos & & $\begin{array}{l}\text { Blackfoot, } \\
\text { Crow }\end{array}$ & $\begin{array}{l}0531562 \\
4858602\end{array}$ & 6823 & $\begin{array}{r}\text { N43.52.790 } \\
\text { W110.36.427 }\end{array}$ \\
\hline & & Chimaphila & umbellata & $\begin{array}{l}\text { Western } \\
\text { Plains }\end{array}$ & $\begin{array}{l}0528792 \\
4865380\end{array}$ & 6880 & $\begin{array}{r}\text { N43.94.099 } \\
\text { W110.64.126 } \\
\end{array}$ \\
\hline & & Pyrola & & Blackfoot & $\begin{array}{l}0532830 \\
4843844\end{array}$ & 7052 & $\begin{array}{r}\mathrm{N} 43.44 .813 \\
\text { W110.35.535 }\end{array}$ \\
\hline & & Vaccinium & & $\begin{array}{l}\text { Western } \\
\text { Plains }\end{array}$ & $\begin{array}{l}0528878 \\
4865473 \\
\end{array}$ & 6805 & $\begin{array}{r}\mathrm{N} 43.56 .507 \\
\mathrm{~W} 110.38 .409\end{array}$ \\
\hline & & Vaccinium & & $\begin{array}{l}\text { Western } \\
\text { Plains }\end{array}$ & $\begin{array}{l}0532757 \\
4855546 \\
\end{array}$ & 7478 & $\begin{array}{r}\mathrm{N} 43.46 .802 \\
\mathrm{~W} 110.26 .147 \\
\end{array}$ \\
\hline 18 & Fabaceae & Lupinus & & $\begin{array}{l}\text { Blackfoot, } \\
\text { Shoshoni }\end{array}$ & $\begin{array}{l}0528887 \\
4865256\end{array}$ & 6885 & $\begin{array}{r}\text { N43.56.391 } \\
\text { W110.38.403 }\end{array}$ \\
\hline 19 & Gentianaceae & Frasera & speciosa & $\begin{array}{l}\text { Arapaho, } \\
\text { Shoshoni }\end{array}$ & $\begin{array}{l}0535819 \\
4846838 \\
\end{array}$ & 6778 & $\begin{array}{r}\text { N43.46.423 } \\
\text { W110.33.294 } \\
\end{array}$ \\
\hline 20 & Geraniaceae & Geranium & viscosissium & Blackfoot & $\begin{array}{l}0528771 \\
4865202 \\
\end{array}$ & 6831 & $\begin{array}{r}\text { N43.56.364 } \\
\text { W110.38.495 } \\
\end{array}$ \\
\hline 21 & Grossulariaceae & Ribes & & $\begin{array}{l}\text { Blackfoot, } \\
\text { Shoshoni. } \\
\text { Western } \\
\text { Plains }\end{array}$ & $\begin{array}{l}0530112 \\
4845419\end{array}$ & 6658 & $\begin{array}{r}\text { N43.45.675 } \\
\text { W110.37.560 } \\
\end{array}$ \\
\hline & & Ribes & $\begin{array}{l}\text { cereum var. } \\
\text { pedicellare }\end{array}$ & $\begin{array}{l}\text { Blackffoot, } \\
\text { Shoshoni. } \\
\text { Western } \\
\text { Plains } \\
\end{array}$ & $\begin{array}{l}0524383 \\
4834094 \\
\end{array}$ & 6550 & $\begin{array}{r}\mathrm{N} 43.39 .57 \\
\mathrm{~W} 110.41 .870 \\
\end{array}$ \\
\hline 22 & Hyacinthaceae & Camassia & quamash & Shoshoni & $\begin{array}{l}0528026 \\
4868170 \\
\end{array}$ & 6869 & $\begin{array}{r}\text { N43.58.510 } \\
W 110.39 .037 \\
\end{array}$ \\
\hline 23 & Hydrophyllaceae & Phaecelia & & Shoshoni & $\begin{array}{l}0535152 \\
4860006 \\
\end{array}$ & 6888 & $\begin{array}{r}\mathrm{N} 43.53 .540 \\
\mathrm{~W} 110.33 .741 \\
\end{array}$ \\
\hline 24 & Lamiaceae & Agastache & & Shoshoni & $\begin{array}{l}0528769 \\
4865203 \\
\end{array}$ & 6870 & $\begin{array}{r}\text { N43.56.363 } \\
\text { W110.38.492 } \\
\end{array}$ \\
\hline & & Menthe & & $\begin{array}{l}\text { Blackfoot, } \\
\text { Shoshoni }\end{array}$ & $\begin{array}{l}0532757 \\
4855546 \\
\end{array}$ & 7478 & $\begin{array}{r}\text { N43.46.802 } \\
\text { W110.26.147 }\end{array}$ \\
\hline & & Mentho & & $\begin{array}{l}\text { Blackfoot, } \\
\text { Shoshoni }\end{array}$ & $\begin{array}{l}0533261 \\
4835858 \\
\end{array}$ & 7086 & $\begin{array}{r}\text { N43.40.498 } \\
\text { W110.35.242 } \\
\end{array}$ \\
\hline 25 & Linaceae & Linum & lewisii & Shoshoni & $\begin{array}{r}0540710 \\
4858305 \\
\end{array}$ & 7127 & $\begin{array}{r}\mathrm{N} 43.52 .595 \\
\mathrm{~W} 110.29 .593 \\
\end{array}$ \\
\hline 26 & Melanthiaceae & Zigadenus & $\begin{array}{l}\text { venenosusvar. } \\
\text { Gramineus }\end{array}$ & $\begin{array}{l}\text { Blackfoot, } \\
\text { Shoshoni }\end{array}$ & $\begin{array}{l}0524383 \\
4834094 \\
\end{array}$ & 6550 & $\begin{array}{r}\text { N43.39.57 } \\
W 110.41 .870 \\
\end{array}$ \\
\hline 27 & Nymphaeaceae & Nuphar & polysepala & $\begin{array}{l}\text { Rocky Mtn } \\
\text { \& Western } \\
\text { Plains }\end{array}$ & $\begin{array}{l}0531210 \\
4852198 \\
\end{array}$ & 6907 & $\begin{array}{r}\mathrm{N} 43.49 .330 \\
\mathrm{~W} 110.36 .713 \\
\end{array}$ \\
\hline 28 & Onagraceae & Chamerion & & Blackfoot & $\begin{array}{l}0533261 \\
4835858 \\
\end{array}$ & 7086 & $\begin{array}{r}\mathrm{N} 43.40 .498 \\
\mathrm{~W} 110.35 .242 \\
\end{array}$ \\
\hline 29 & Orchidaceae & Corallorhiza & & Shoshoni & $\begin{array}{l}0532757 \\
4855546\end{array}$ & 7478 & $\begin{array}{r}\text { N43.46.802 } \\
\text { W110.26.147 } \\
\end{array}$ \\
\hline 30 & Pinaceac & Abies & lasiocarpa & $\begin{array}{l}\text { Blackfoot, } \\
\text { Crow, } \\
\text { Shoshoni }\end{array}$ & $\begin{array}{l}0528887 \\
4865256 \\
\end{array}$ & 6885 & $\begin{array}{r}\mathrm{N} 43.56 .391 \\
\mathrm{~W} 110.38 .403 \\
\end{array}$ \\
\hline & & Pinus & contoria & Plains & $\begin{array}{l}528792 \\
486580 \\
\end{array}$ & 6880 & $\begin{array}{r}\text { N43.94.099 } \\
\text { W110.64.126 } \\
\end{array}$ \\
\hline & & Pinus & flexilis & $\begin{array}{l}\text { Westem } \\
\text { Plains }\end{array}$ & $\begin{array}{l}0523901 \\
4848535 \\
\end{array}$ & 6996 & $\begin{array}{r}\text { N43.47.371 } \\
\text { W110.42.177 } \\
\end{array}$ \\
\hline & & Psendotsuga & & Blackfoot & $\begin{array}{l}0542112 \\
4858508 \\
\end{array}$ & 7010 & $\begin{array}{r}\mathrm{N} 43.52 .706 \\
\mathrm{~W} 110.28 .549 \\
\end{array}$ \\
\hline & & Pseudotsuga & & Blackfoot & $\begin{array}{l}0532830 \\
4843844 \\
\end{array}$ & 7052 & $\begin{array}{r}\mathrm{N} 43.44 .813 \\
\mathrm{~W} 110.35 .535 \\
\end{array}$ \\
\hline 31 & Poaceae & Hesperostipa & comata & Blackfoot & $\begin{array}{l}0535433 \\
4856587 \\
\end{array}$ & 6733 & $\begin{array}{r}\text { N43.51.646 } \\
\text { W110.33.506 } \\
\end{array}$ \\
\hline 32 & Polygonaceae & Rumex & & $\begin{array}{l}\text { Arapaho, } \\
\text { Blackfoot, } \\
\text { Shoshoni }\end{array}$ & $\begin{array}{l}0535378 \\
4856723 \\
\end{array}$ & 6774 & $\begin{array}{r}\text { N43.51.764 } \\
\text { W110.33.584 } \\
\end{array}$ \\
\hline 33 & Ranunculaceae & Aconitum & columbianum & Blackfoot & $\begin{array}{l}0517616 \\
4885204\end{array}$ & 7164 & $\begin{array}{r}\text { N44.07.180 } \\
\text { W110.46.794 } \\
\end{array}$ \\
\hline & & Actaea & & $\begin{array}{l}\text { Blackfoot, } \\
\text { Plains }\end{array}$ & $\begin{array}{l}0540619 \\
4858956 \\
\end{array}$ & 7127 & $\begin{array}{r}\text { N43.52.955 } \\
\text { W11029.661 } \\
\end{array}$ \\
\hline & & Aquilegia & & Shoshoni & $\begin{array}{l}0544999 \\
4847335 \\
\end{array}$ & 7454 & $\begin{array}{r}\text { N43.46.665 } \\
\text { W110.26.448 } \\
\end{array}$ \\
\hline & & Delphinium & bicolor & $\begin{array}{l}\text { Shoshoni, } \\
\text { Blackfoot }\end{array}$ & $\begin{array}{l}0528792 \\
4865380 \\
\end{array}$ & 6880 & $\begin{array}{r}\text { N43.94.099 } \\
\text { W110.64.126 } \\
\end{array}$ \\
\hline & & Thalictrum & & $\begin{array}{l}\text { Shoshoni, } \\
\text { Blackfoot }\end{array}$ & $\begin{array}{l}0528878 \\
4865473\end{array}$ & 6805 & $\begin{array}{r}\text { N43.56.507 } \\
\text { W110.38.409 } \\
\end{array}$ \\
\hline 34 & Rosaceae & Amelanchier & & $\begin{array}{l}\text { Shoshoni, } \\
\text { Blackfoot }\end{array}$ & $\begin{array}{l}0528607 \\
4865237 \\
\end{array}$ & 6872 & $\begin{array}{r}\text { N43.56.383 } \\
\text { W110.38.616 }\end{array}$ \\
\hline & & Amelanchier & & $\begin{array}{l}\text { Shoshoni, } \\
\text { Blackfoot }\end{array}$ & $\begin{array}{l}0525682 \\
4830442 \\
\end{array}$ & 6684 & $\begin{array}{r}\text { N43.37.592 } \\
\text { W110.40.899 } \\
\end{array}$ \\
\hline & & Ceanothus & velutinous & $\begin{array}{l}\text { Arapaho, } \\
\text { Shoshoni }\end{array}$ & $\begin{array}{r}0528853 \\
4865128 \\
\end{array}$ & 6875 & $\begin{array}{r}\text { N43.56.322 } \\
\text { W110.38.432 } \\
\end{array}$ \\
\hline & & Fragaria & & $\begin{array}{l}\text { Blackfoot, } \\
\text { Shoshoni }\end{array}$ & $\begin{array}{r}0542112 \\
4858508 \\
\end{array}$ & 7010 & $\begin{array}{r}\text { N43.52.706 } \\
\text { W110.28.549 } \\
\end{array}$ \\
\hline & & Geum & triflorum & Blackfoot & $\begin{array}{l}0524383 \\
4834094 \\
\end{array}$ & 6550 & $\begin{array}{r}\text { N43.39.57 } \\
\text { W } 110.41 .870 \\
\end{array}$ \\
\hline & & Geum & & Blackfoot & $\begin{array}{l}0524383 \\
4834094 \\
\end{array}$ & 6550 & $\begin{array}{r}\text { N43.39.57 } \\
\text { W } 110.41 .870 \\
\end{array}$ \\
\hline & & Potentilla & & $\begin{array}{l}\text { Blackfoot, } \\
\text { Gosiute }\end{array}$ & $\begin{array}{l}0504300 \\
4852900 \\
\end{array}$ & 6767 & $\begin{array}{r}\text { N43.49.690 } \\
\text { W110.29.927 } \\
\end{array}$ \\
\hline & & Prunus & virginiana & $\begin{array}{l}\text { Blackfoot, } \\
\text { Crow, } \\
\text { Shoshoni, } \\
\text { Plains } \\
\end{array}$ & $\begin{array}{l}0528792 \\
4865380 \\
\end{array}$ & 6880 & $\begin{array}{r}\text { N43.94.099 } \\
\text { W110.64.126 } \\
\end{array}$ \\
\hline & & Purshia & tridentata & Shoshoni & $\begin{array}{l}0530112 \\
4845419 \\
\end{array}$ & 6658 & $\begin{array}{r}\text { N43.45.675 } \\
\text { W110.37.560 } \\
\end{array}$ \\
\hline & & Purshia & tridentata & Shoshoni & $\begin{array}{l}0531210 \\
4852198 \\
\end{array}$ & 6907 & $\begin{array}{r}\mathrm{N} 43.49 .330 \\
\text { W110.36.713 } \\
\end{array}$ \\
\hline & & Rosa & woodsii & $\begin{array}{l}\text { Arapaho, } \\
\text { Blackfoot, } \\
\text { Crow, } \\
\text { Shoshoni }\end{array}$ & $\begin{array}{l}0504300 \\
4852900 \\
\end{array}$ & 6767 & $\begin{array}{r}\text { N43.49.690 } \\
\text { W110.29.927 }\end{array}$ \\
\hline & & Rubus & parviflorus & Blackfoot & $\begin{array}{l}0540619 \\
4858956 \\
\end{array}$ & 7127 & $\begin{array}{r}\text { N43.52.955 } \\
\text { W11029.661 } \\
\end{array}$ \\
\hline 35 & Salicaceae & Salix & & $\begin{array}{l}\text { Blackfoot, } \\
\text { Crow, }\end{array}$ & $\begin{array}{l}0535805 \\
4856073 \\
\end{array}$ & 6773 & $\begin{array}{r}\mathrm{N} 43.51 .411 \\
\mathrm{~W} 110.33 .267 \\
\end{array}$ \\
\hline
\end{tabular}




\begin{tabular}{|c|c|c|c|c|c|c|c|}
\hline & & & & Shoshoni & & & \\
\hline \multirow[t]{4}{*}{36} & Scrophulariaceae & Castelleja & & $\begin{array}{l}\text { Blackfoot, } \\
\text { Shoshoni }\end{array}$ & $\begin{array}{l}0528853 \\
4865128 \\
\end{array}$ & 6875 & $\begin{array}{r}\mathrm{N} 43.56 .322 \\
\text { W110.38.432 }\end{array}$ \\
\hline & & Mimulus & & Shoshoni & $\begin{array}{l}0517164 \\
4885688 \\
\end{array}$ & 7301 & $\begin{array}{r}\text { N44.07.457 } \\
\text { W110.47.135 }\end{array}$ \\
\hline & & Pedicularis & groenlandica & & $\begin{array}{l}0531562 \\
4858602 \\
\end{array}$ & 6823 & $\begin{array}{r}\text { N43.52.790 } \\
\text { W110.36.427 }\end{array}$ \\
\hline & & Pedicularis & groenlandica & & $\begin{array}{l}0531562 \\
4858602 \\
\end{array}$ & 6823 & $\begin{array}{r}\mathrm{N} 43.52 .790 \\
\text { W110.36.427 }\end{array}$ \\
\hline 37 & Typhaceae & Typha & & $\begin{array}{l}\text { Backfoot, } \\
\text { Rocky } \\
\text { Mountain } \\
\end{array}$ & $\begin{array}{l}0526333 \\
4871225 \\
\end{array}$ & 6940 & $\begin{array}{r}\mathrm{N} 43.59 .622 \\
\text { W11.0.40.296 }\end{array}$ \\
\hline 38 & Urticaceae & Urtica & & Shoshoni & $\begin{array}{l}0540619 \\
4858956 \\
\end{array}$ & 7127 & $\begin{array}{r}\text { N43.52.955 } \\
\text { W11029.661 } \\
\end{array}$ \\
\hline \multirow[t]{2}{*}{39} & Valerianaceae & Valeriana & edulis & Blackfoot & $\begin{array}{l}0540710 \\
4858305 \\
\end{array}$ & 7127 & $\begin{array}{r}\mathrm{N} 43.52 .595 \\
\text { W110.29.593 }\end{array}$ \\
\hline & & Valeriana & edulis & Blackfoot & $\begin{array}{l}0524546 \\
4882455 \\
\end{array}$ & 6830 & $\begin{array}{r}\text { N44. } 05.698 \\
\text { W110.41.602 }\end{array}$ \\
\hline
\end{tabular}

